\title{
Efeito residual da gessagem e calagem na resistência à penetração do solo obtida com dois penetrômetros
}

\section{Marcela Pacola Oliveira ${ }^{1}$, Cassiano Garcia Roque ${ }^{1}$, Monica Cristina Rezende Zuffo Borges ${ }^{1}$, Roberta Pacola Oliveira ${ }^{1}$, Kenio Batista Nogueira ${ }^{1}$}

\author{
${ }^{1}$ Universidade Federal de Mato Grosso do Sul, Campus de Chapadão do Sul, Chapadão do Sul, Brasil. E-mail: \\ marcela_pacola@hotmail.com, cassiano.roque@ufms.br, monica.zuffo-borges@hotmail.com, robertapacola@hotmail.com, \\ kenio.nogueira@ufms.br
}

Recebido: 02/08/2016; Aceito: 19/02/2017.

\section{RESUMO}

A resistência do solo à penetração é um dos fatores limitantes para o desenvolvimento das culturas. O objetivo foi avaliar o efeito residual de gessagem e calagem na resistência à penetração medida com o penetrômetro de impacto e o eletrônico em sistema de semeadura direta e convencional, em diferentes sistemas de condicionamento do solo. O delineamento utilizado foi de blocos casualizados com os fatores arranjados em parcelas subdivididas. As parcelas receberam as aplicações de calcário e gesso agrícola (controle, calcário (3,563 $\left.\mathrm{Mg} \mathrm{ha}^{-1}\right)$, gesso $\left(2 \mathrm{Mg} \mathrm{ha}^{-1}\right)$, calcário dose recomendada + gesso $\left(3,563 \mathrm{Mg} \mathrm{ha}^{-1}+2 \mathrm{Mg} \mathrm{ha}^{-1}\right)$, calcário metade da dose recomendada + gesso $\left(1,781 \mathrm{Mg} \mathrm{ha}^{-1}+2 \mathrm{Mg} \mathrm{ha}^{-1}\right)$ e calcário dobro da dose recomendada + gesso $(7,126 \mathrm{Mg}$ $\left.\mathrm{ha}^{-1}+2 \mathrm{Mg} \mathrm{ha}^{-1}\right)$, com quatro repetições e as subparcelas as profundidades $(0,10-0,20 ; 0,20-0,30$ e 0,30-0,40 m). A resistência à penetração não foi influenciada pelo sistema de condicionamento do solo. Ambos os penetrômetros "impacto e eletrônico" podem ser utilizados para obtenção da resistência à penetração.

Palavras-chave: Compactação; Plantio direto; Plantio convencional.

\section{Residual effect of gypsum and liming on soil resistance to penetration obtained with two penetrometer}

\begin{abstract}
Soil resistance to penetration is one of the limiting factors for crop development, becoming a mechanical impediment to the growth of roots. The study was conducted in Chapadao do Sul, MS, Brazil and aimed to evaluate and compare the soil resistance to penetration measured by the impact penetrometer and electronic in an Oxisoil under different management systems (no-till and conventional system) with application of soil conditioning. The experiment was arranged in a randomized block design with the factors arranged in a split plot. The plots received the applications of lime and gypsum (control, limestone (3,563 $\left.\mathrm{Mg} \mathrm{ha}^{-1}\right)$, gypsum $\left(2 \mathrm{Mg} \mathrm{ha}^{-1}\right)$, lime recommended rate + gypsum $\left(3,563 \mathrm{Mg} \mathrm{ha}^{-1}+2 \mathrm{Mg} \mathrm{ha}^{-1}\right)$, lime half the recommended rate + gypsum $(1,781$ $\left.\mathrm{Mg} \mathrm{ha}^{-1}+2 \mathrm{Mg} \mathrm{ha}^{-1}\right)$ and lime twice the recommended rate + gypsum $\left(7,126 \mathrm{Mg} \mathrm{ha}^{-1}+2 \mathrm{Mg} \mathrm{ha}^{-1}\right)$, with four replicates and subplots depths $(0.10-0.20 ; 0.20-0.30$ and $0.30-0.40 \mathrm{~m})$. The penetration resistance was not influenced by soil conditioning system, after three years of experimental implementation. The impact penetrometer presented superior values of resistance to penetration, but the impact penetrometer was efficient to characterize the compression. There was positive correlation between the average penetrometers for both no-till and conventional tillage.
\end{abstract}

Key words: Compression; No till; conventional tillage. 


\section{Introdução}

Nos solos do cerrado, a acidez é um dos problemas limitantes para produção e desenvolvimento das culturas, desta maneira, a correção da fertilidade do solo torna-se uma prática indispensável. A calagem é entre as práticas de correção do solo mais eficiente para elevar o pH, a saturação por bases e os teores de Ca, e diminuir Al e Mn trocáveis no solo (CAIRES et al., 2004). A reação do calcário está restringida ao local de aplicação, consequentemente, não possui efeito eficaz na acidez do subsolo, dependendo da lixiviação de sais através do perfil (CAIRES et al., 2004). Desta foram vários trabalhos destacam a prática e eficiência da aplicação de gesso no subsolo (ROCHA et al., 2008; SANTOS et al., 2013).

A resistência do solo à penetração é um dos fatores limitantes para o desenvolvimento das culturas, torna-se um impedimento mecânico para o crescimento das raízes afetando processos químicos e biológicos do solo além de interferir nos processos de condutividade ao ar, aeração, à água e ao calor, infiltração e redistribuição da água no solo (CAMARGO; ALLEONI, 1997).

Uma das opções de manejo de solo é o sistema de semeadura direta, utilizado para produção de grãos ou integrado à pecuária, porém há aumento dos problemas relacionados à compactação pelo uso de maquinários pesados e o não revolvimento do solo (MAZURANA et al., 2011).

O sistema radicular do milho não se desenvolve corretamente em áreas que apresentam estruturas compactadas. De acordo com Arshad et al., (1996), valores de RP que variam de 2,0 a 4,0 Mpa restringem ou impedem o desenvolvimento das raízes. Foloni et al., (2003) em trabalho semelhante, observaram que o valor de RP de 1,4 Mpa impede o crescimento do sistema radicular do milho, interferindo negativamente na sua produtividade. Freddi et al., (2006), ao estudarem a cultura do milho, apontaram que solos com valores entre 0,9 e 2,0 Mpa não restringiram a produtividade.

É importante conhecer as condições do solo e determinar sua compactação haja visto que a mesma poderá causar impedimento mecânico à penetração das raízes das plantas. Desta forma, existem equipamentos denominados "penetrômetros" que são utilizados para verificar, caso houver, uma camada compactada no solo. $\mathrm{O}$ equipamento determina a resistência que o solo oferece à algo que tente se movimentar através dele tais como as raízes das plantas (PEDROTTI et al., 2001).

Com a diversidade de penetrômetros existentes para determinação de resistência à penetração, é importante que se conheça a proximidade dos resultados obtidos, para que haja melhor interpretação dos dados. Um dos equipamentos mais utilizados para determinação da compactação é o penetrômetro de impacto, que segundo Tormena \& Roloff (1996) não possui necessidade de calibração, tem baixo custo e não sofre interferência do operador. Outro equipamento que vem sendo amplamente difundido e utilizado é o penetrômetro estático eletrônico por sua praticidade e rapidez, porém segundo Herrick \& Jones (2002), apesar da necessidade de constância do operador estes equipamentos apresentam um método mais padronizado.

Este trabalho objetivou avaliar o efeito residual da calagem e gessagem na resistência à penetração em sistema de semeadura direta e convencional, obtida por penetrômetro de impacto e eletrônico.

\section{Material e Métodos}

O experimento foi conduzido na área experimental da Universidade Federal de Mato Grosso do Sul, Município de Chapadão do Sul, Estado de Mato Grosso do Sul, sendo as coordenadas geográficas $18^{\circ} 46^{\prime} 44^{\prime \prime} \mathrm{S}$ e 52 36' 59" W e altitude de $810 \mathrm{~m}$ em um Latossolo Vermelho distrófico (LVd), textura argilosa. O clima é do tipo tropical úmido (Aw), com estação chuvosa no verão e seca no inverno, com temperatura média anual variando de $13{ }^{\circ} \mathrm{C}$ a $28{ }^{\circ} \mathrm{C}$, e precipitação média anual de $1.850 \mathrm{~mm}$ e umidade relativa média anual de $64,8 \%$ (CASTRO et al., 2012).

Em julho de 2011, realizou-se amostragens de solos para caracterização química e física antes da instalação do experimento. As amostras químicas foram retiradas antes da instalação do experimento nas profundidades de 0,0-0,20 e 0,20-0,40 m, coletando-se dez amostras simples aleatórias de solo para compor a amostra composta (Tabela 1).

Tabela 1. Atributos químicos do solo da área experimental antes da instalação do experimento, coletadas em duas profundidades. (Chapadão do Sul, MS, 2011)

\begin{tabular}{|c|c|c|c|c|c|c|c|c|c|c|c|c|c|}
\hline Prof. & $\mathrm{pH}$ & $\mathrm{P}$ & $\mathrm{K}$ & $\mathrm{Ca}$ & $\mathrm{Mg}$ & $\mathrm{H}+\mathrm{Al}^{1}$ & $\mathrm{CTC}^{2}$ & $\mathrm{Al}$ & $\mathrm{SB}^{3}$ & $\mathrm{t}^{4}$ & $\mathbf{M}^{5}$ & $\mathrm{~V}^{6}$ & $\mathrm{MO}^{7}$ \\
\hline $\mathrm{m}$ & $\left.\mathrm{Ca} \mathrm{Cl}_{2}\right)$ & \multicolumn{3}{|c|}{----mg dm³ } & \multicolumn{6}{|c|}{ 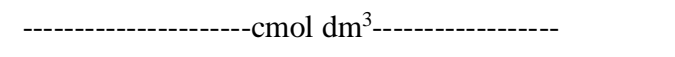 } & \multicolumn{2}{|c|}{----\%--- } & $\mathrm{g} \mathrm{kg}^{-1}$ \\
\hline $0,0-0,20$ & 4,5 & 11,1 & 109,48 & 2,7 & 1,0 & 5,0 & 9,0 & 0,07 & 3,98 & 4,05 & 1,7 & 44,3 & 37,7 \\
\hline $0,20-0,4$ & 1,8 & 3,6 & 74,29 & 1,7 & 0,6 & 5,5 & 8,0 & 0,14 & 2,49 & 2,63 & 5,3 & 31,2 & 24,6 \\
\hline
\end{tabular}


A preparação da área experimental iniciou em 10 de outubro de 2011 com a aplicação e incorporação de calcário em área total, sendo a profundidade de trabalho do escarificador de $0,25 \mathrm{~m}$. Após uma semana, foi realizada aplicação superficial de gesso agrícola em área total, seguida de gradagem no solo, tanto no sistema semeadura convencional (SSC) quanto no sistema de semeadura direta (SSD), antes da semeadura da primeira sucessão soja/milho. No condicionamento químico do solo, utilizou-se calcário dolomítico com $23,3 \%$ de $\mathrm{CaO}, 17,5 \%$ de $\mathrm{MgO}$ e $75 \%$ de Poder Relativo de Neutralização Total (PRNT) e gesso agrícola com $18,63 \%$ de cálcio e $15,70 \%$ de enxofre. A dose de calcário adotada teve o objetivo de elevar a saturação por bases para $74 \%$ na camada de $0,0-0,20 \mathrm{~m}$, uma vez que há certa tendência pelos produtores rurais da região dos chapadões a utilizar doses maiores de calcário a fim de aumentar a saturação por bases para mais de $70 \%$. Isto ocorre, principalmente, na correção do solo na implantação do sistema de semeadura direta, como é o caso deste trabalho. Para o cálculo do gesso utilizou-se a porcentagem de argila no solo (Tabela 2).

Tabela 2. Caracterização granulométrica. (Chapadão do Sul, MS, 2011).

\begin{tabular}{|c|c|c|c|}
\hline \multirow{2}{*}{$\begin{array}{c}\text { Frações do Solo } \\
\left(\mathrm{g} \mathrm{kg}^{-1}\right)\end{array}$} & \multicolumn{3}{|c|}{ Profundidade (m) } \\
\hline & $0,0-0,10$ & $0,10-0,20$ & $0,20-0,30$ \\
\hline Argila & 400 & 400 & 400 \\
\hline Silte & 100 & 100 & 95 \\
\hline Areia & 500 & 500 & 505 \\
\hline
\end{tabular}

O milho segunda safra foi cultivado no sistema de semeadura direta (SSD) e sistema semeadura convencional (SSC). O SSC consistiu do preparo do solo antes da instalação da cultura, realizado anualmente sempre com o uso de grade intermediária com 18 discos de 28 polegadas para o preparo primário e de grade niveladora com 28 discos de 20 polegadas para o preparo secundário.

Os sistemas de preparo foram avaliados de forma independente, no qual o delineamento experimental utilizado foi o de blocos casualizados, arranjado em esquema de parcelas subdivididas, com quatro repetições distribuídas em parcelas de 5,0 x 5,0 m, sendo a parcela útil constituída de 3 linhas centrais de 3 metros de comprimento. Consideram-se como parcelas as doses com os seguintes tratamentos: controle, calcário $\left(3,563 \mathrm{Mg} \mathrm{ha}^{-1}\right)$, gesso $\left(2 \mathrm{Mg} \mathrm{ha}^{-1}\right)$, calcário dose recomendada + gesso $\left(3,563 \mathrm{Mg} \mathrm{ha}^{-1}+2 \mathrm{Mg} \mathrm{ha}^{-1}\right)$, calcário metade da dose recomendada + gesso $(1,781$ $\left.\mathrm{Mg} \mathrm{ha}^{-1}+2 \mathrm{Mg} \mathrm{ha}^{-1}\right)$ e calcário dobro da dose recomendada + gesso $\left(7,126 \mathrm{Mg} \mathrm{ha}^{-1}+2 \mathrm{Mg} \mathrm{ha}^{-1}\right)$ e as subparcelas as três profundidades: $0,10-0,20 ; 0,20-0,30$ e 0,30-0,40 m, assim tem-se 24 parcelas por sistema de preparo.
As amostras foram coletadas aos 30 meses após a aplicação do calcário e do gesso, nas camadas de 0,10 $0,0,20 ; 0,20-0,30$ e $0,30-0,40 \mathrm{~m}$ de profundidade, na área útil de cada parcela, coletadas sempre na entrelinha da cultura do milho, dentro de cada sistema de cultivo SSC e SSD.

Para a determinação da resistência mecânica do solo à penetração (RP), utilizou-se o penetrômetro de impactos (PI) modelo IAA/Planalsucar-Stolf.

Outro equipamento utilizado para determinar a resistência à penetração foi o penetrômetro eletrônico digital (PE) de dados PLG 1020 penetroLOG, da marca Falker. Foram amostrados três pontos por parcela. A obtenção dos dados deste equipamento é feita por esforço manual, onde a ponta cônica e a haste do equipamento foram inseridas no solo por meio da força do operador. Para o processamento dos dados de resistência à penetração, foi utilizado o Software PenetroLOG. Os dados de RP foram classificados a cada $0,10 \mathrm{~m}$ até a profundidade de $0,40 \mathrm{~m}$, apresentados em KPa e posteriormente convertidos para MPa.

A amostra deformada do solo para determinação da umidade volumétrica (UV), foi coletada nas diferentes profundidades de $0,10-0,20,0,20-0,30$ e $0,30-0,40 \mathrm{~m}$, no mesmo momento do teste de resistência mecânica à penetração, utilizando-se um trado do tipo holandês. Os dados foram avaliados pelo teste de Tukey a $1 \%$ de probabilidade para comparação de médias, para a análise estatística foi utilizado o software Assistat

\section{Resultados e Discussão}

Na Tabela 3 são apresentados os resultados da análise de variância de resistência à penetração obtidos pelo Penetrômetro de Impacto (PI). Verifica-se que para profundidade houve diferença significativa tanto para SSC quanto para SSD, corroborando com Carvalho et al. (2012). Não houve diferença estatística na resistência à penetração para os sistemas de condicionamento de solo, em ambos os sistemas de preparo.

Tabela 3. Análise de variância dos resultados obtidos para resistência mecânica à penetração (RP) com penetrômetro de impacto (PI) em diferentes sistemas de condicionamento do solo e profundidade. (Chapadão do Sul, MS, 2015).

\begin{tabular}{ccc}
\hline Fonte de & \multicolumn{2}{c}{ Valor $-\mathrm{p}$} \\
\cline { 2 - 3 } variação & SSD & SSC \\
\hline SCS & $0,08^{\text {ns }}$ & $1,29^{\text {ns }}$ \\
P & $13,46^{* *}$ & $12,55^{* *}$ \\
SCS $* P$ & $0,47^{\text {ns }}$ & $1,14^{\text {ns }}$ \\
\hline CVSCS & 38,54 & 14,54 \\
CVP & 12,41 & 11,08 \\
\hline
\end{tabular}

SCS: sistema de condicionamento do solo; P: Profundidade; SCSxP: Interação entre sistema de manejo x Profundidade; CVP: coeficiente de variação de profundidade (\%) e CVSCS: coeficiente de variação de sistema de condicionamento do solo (\%).ns, **: Não significativo e significativo respectivamente a $1 \%$ pelo teste $\mathrm{F}$. 
O mesmo resultado não foi obtido por Müller et al. (2012), cujo estudo versou sobre os efeitos de doses de gesso aplicados na superfície sobre a qualidade estrutural do solo de um Latossolo Vermelho após 50 meses de aplicação em sistema de semeadura direta. Os autores obtiveram diferença para resistência à penetração (RP) nas camadas 0,075-0,30 m de profundidade, justificando esta diferença pelo aumento de agregados em consequência do aumento de teor $\mathrm{Ca}$ na subsuperfície o que aumenta a força de agregação das partículas, porém estes valores não foram suficientes para restringirem o crescimento radicular.

Independente dos sistemas de preparo, a camada de 0,20-0,30 $\mathrm{m}$ apresenta os maiores valores de compactação, sendo que no SSD não diferiu da camada de 0,10 - 0,20 m (Tabela 4). A umidade volumétrica para o SSD não apresentou diferença, não interferindo na compactação do solo. Já no SSC, houve diferença na umidade do solo, onde a camada mais profunda obteve maior umidade volumétrica.

No sistema de SSD a camada de 0,10-0,20 m não diferiu estaticamente da camada 0,20-0,30 m apresentando os maiores valores de RP e UV em relação ao SSC, o que favorece a biodiversidade, proporcionando assim uma melhoria nas propriedades físicas e químicas do solo. Em trabalhos desenvolvidos, Tavares Filho et al. (2001) obtiveram maiores valores de RP em camada superficial do solo (0,0-0,15 m e 0,0$0,20 \mathrm{~m})$, porém não foram suficientes para interferirem no crescimento radicular.

No SSC as camadas que obtiveram menor resistência à penetração foram a $0,10-0,20 \mathrm{~m}$ e 0,30 $0,40 \mathrm{~m}$. A camada $0,20-0,30 \mathrm{~m}$ apresentou maiores valores de RP e menor UV, fato este que pode ser explicado pelo acúmulo de cargas dos implementos que foram utilizados no preparo do solo, indicando que houve a formação de "pé de grade"; resultados semelhantes foram encontrados por Raslich et al. (2008).

Na Tabela 5 são apresentados os resultados da análise de variância da resistência mecânica, obtida pelo penetrômetro eletrônico, em diferentes sistemas de condicionamento do solo (SCS), profundidade (P) e sistemas de preparo. Observa-se que houve diferença significativa para as diferentes profundidades, tanto para SSD quanto para SSC, resultados estes que foram obtidos também com o penetrômetro de impacto.

Verificou-se na Tabela 6, que as médias de resistência à penetração, em $\mathrm{MPa}$, obtidas na camada de 0,20-0,30 m em ambos os sistemas de preparo do solo foram maiores. Resultados que foram obtidos também com o penetrômetro de Impacto.

No SSD observa-se uma tendência, apesar da não diferença estatística para a camada de $0,30-0,40 \mathrm{~m}$ à uma menor RP na camada de 0,10 - 0,20 m. Este fato pode ser explicado pelo sistema radicular fasciculado da cultura do milho, pois tratando-se de uma gramínea, suas raízes se concentram nas camadas superficiais do solo. Em estudo, os autores Tavares Filho et al., (2001), constataram que valores acima de 3,5 Mpa não restringem o desenvolvimento radicular do milho. Os valores alcançados de 1,95, 2,33 e 2,06 MPa $\mathrm{r}$ para as profundidades de 0,10-0,20 m, 0,20-0,30 m e 0,30-0,40 $\mathrm{m}$ respectivamente em Latossolo Vermelho Distrófico ficaram abaixo dos valores críticos de resistência mecânica à penetração para o desenvolvimento da cultura. Resultados semelhantes foram obtidos por Freddi et al. (2006).

Tabela 5. Análise de variância dos resultados obtidos para resistência mecânica à penetração (RP) com o penetrômetro eletrônico em diferentes sistemas de condicionamento do solo e profundidade. (Chapadão do Sul, MS, Brasil, 2015).

\begin{tabular}{lcc}
\hline Fonte de variação & \multicolumn{2}{c}{ Valor $-\mathrm{p}$} \\
\cline { 2 - 3 } & $\mathrm{SSD}$ & $\mathrm{SSC}$ \\
\hline SCS & $0,10^{\mathrm{ns}}$ & $1,73^{\mathrm{ns}}$ \\
$\mathrm{P}$ & $10,24^{* *}$ & $7,55^{* *}$ \\
SCS $* \mathrm{P}$ & $1,68^{\mathrm{ns}}$ & $1,50^{\mathrm{ns}}$ \\
\hline CVSCS & 20,30 & 19,76 \\
CVP & 14,41 & 16,20 \\
\hline
\end{tabular}

SCS: sistema de condicionamento do solo; P: Profundidade; SCSxP: Interação entre sistema de manejo x Profundidade; CVP: coeficiente de variação de profundidade (\%) e CVSCS: coeficiente de variação de sistema de condicionamento do solo (\%).ns, **: Não significativo e significativo respectivamente a $1 \%$ pelo teste $\mathrm{F}$.

Tabela 4. Valores das médias obtidas com o penetrômetro de impacto nas diferentes profundidades para RP e sistemas de preparo. (Chapadão do Sul, MS, 2015).

\begin{tabular}{ccccc}
\hline & \multicolumn{3}{c}{ SSC } \\
\hline Profundidade & RP & UV & RP & UV \\
\cline { 2 - 5 } & $(\mathrm{MPa})$ & $\left(\mathrm{m}^{3} \mathrm{~m}^{-3}\right)$ & $(\mathrm{MPa})$ & $\left(\mathrm{m}^{3} \mathrm{~m}^{-3}\right)$ \\
\hline $0,10-0,20 \mathrm{~m}$ & $3,13 \mathrm{a}$ & $36,66 \mathrm{a}$ & $2,73 \mathrm{~b}$ & $32,09 \mathrm{~b}$ \\
$0,20-0,30 \mathrm{~m}$ & $3,30 \mathrm{a}$ & $34,27 \mathrm{a}$ & $3,14 \mathrm{a}$ & $29,80 \mathrm{~b}$ \\
$0,30-0,40 \mathrm{~m}$ & $2,74 \mathrm{~b}$ & $34,12 \mathrm{a}$ & $2,76 \mathrm{~b}$ & $36,22 \mathrm{a}$ \\
\hline
\end{tabular}

Médias seguidas de letras iguais minúscula na coluna, não diferem estatisticamente entre si pelo teste de Tukey a 1\%. SSD: Sistema de semeadura direto; SSC: Sistema de semeadura convencional, UV: Umidade volumétrica. 
Tabela 6. Valores das médias obtidas com o penetrômetro eletrônico nas diferentes profundidades para RP e sistemas de preparo. (Chapadão do Sul, MS, 2015).

\begin{tabular}{ccccc}
\hline & \multicolumn{4}{c}{ SSD } \\
\hline \multirow{2}{*}{ Profundidade } & RP & UV & RP & UV \\
\cline { 2 - 5 } & $(\mathrm{MPa})$ & $\left(\mathrm{m}^{3} \mathrm{~m}^{-3}\right)$ & $(\mathrm{MPa})$ & $\left(\mathrm{m}^{3} \mathrm{~m}^{-3}\right)$ \\
\hline $0,10-0,20 \mathrm{~m}$ & $1,95 \mathrm{~b}$ & $36,66 \mathrm{a}$ & $1,85 \mathrm{~b}$ & $32,09 \mathrm{~b}$ \\
$0,20-0,30 \mathrm{~m}$ & $2,33 \mathrm{a}$ & $34,27 \mathrm{a}$ & $2,20 \mathrm{a}$ & $29,80 \mathrm{~b}$ \\
$0,30-0,40 \mathrm{~m}$ & $2,06 \mathrm{~b}$ & $34,12 \mathrm{a}$ & $2,15 \mathrm{a}$ & $36,22 \mathrm{a}$ \\
\hline
\end{tabular}

Médias seguidas de letras iguais minúscula na coluna, não diferem estatisticamente entre si pelo teste de Tukey a 1\%. SSD: Sistema de semeadura direto; SSC: Sistema de semeadura convencional, UV: Umidade volumétrica.

Os resultados obtidos para RP foram superiores com o PI em relação ao PE, em todas as camadas. Resultados semelhantes também foram encontrados por Souza et al., (2014) e Roboredo et al. (2010), que ao estudarem a resistência mecânica do solo em função da umidade e do tipo de penetrômetro em Argissolo Amarelo e em Latossolo Vermelho distrófico, apesar do penetrômetro de impacto ter apresentado maiores valores observaram a presença de camada compactada em ambos os equipamentos.

As camadas mais compactadas no SSC são as mais profundas, $0,20-0,30$ e 0,30-0,40 m; resultado este já esperado uma vez que o uso intensificado de maquinário para o preparo do solo promove a degradação física; os maiores valores de RP encontram-se abaixo da camada trabalhada, ou seja, nas camadas subsuperficiais, indicando o uso de "pé-de-arado" ou "pé- de-grade" (Cortez et al., 2011).

Lima et al., (2013), em estudo realizado, compararam o comportamento da resistência à penetração do solo, de um penetrômetro de impacto e um penetrômetro eletrônico em diferentes profundidades. Os autores observaram que os dois equipamentos obtiveram valores similares em todas as profundidades. Por outro lado, Leite et al., (2010) comparou o penetrômetro eletrônico e manual em uma resistência à penetração já conhecida, obtendo valores diferentes para os equipamentos utilizados; o penetrômetro eletrônico apresentou maior resistência que o penetrômetro manual, sendo mais eficaz para a obtenção do real valor de resistência à penetração.

De acordo com Roboredo et al. (2010), o PI é indicado apenas para a camada $0,00-0,10 \mathrm{~m}$, pois para camadas profundas apresenta restrições de uso. Os autores obtiveram também valores menores pelo $\mathrm{PE}$ devido à velocidade constante da ponteira do equipamento ao penetrar no solo. Assim, torna-se imprescindível o conhecimento sobre o princípio de funcionamento dos equipamentos que serão utilizados para avaliar a compactação do solo.

De acordo com Pacheco e Cantalice (2011), o coeficiente de correlação linear simples, indica que há relação linear entre duas variáveis; a mudança de uma variável implica em mudança constante no valor médio da outra variável. Os penetrômetros apresentaram uma correlação moderada de 0,34 no SSD e 0,33 SSC (Figura 1) indicando que os mesmos conduzem a valores relativamente semelhantes, podendo ser utilizado qualquer um dos equipamentos para obtenção da resistencia à penetração.

Estes resultados corroboram com Roque et al., (2003) e Beutler et al., (2007) que tambem compararam penetrômetros de impacto e estático, de impacto de anel dinamométrico e eletrônico, e de impacto e anel dinamométric, e obtiveram valores semelhantes para os equipamentos.

Finalmente constata-se que o PI apresentou valores superiores em relação ao PE, porém ambos indicaram a camada compactada.
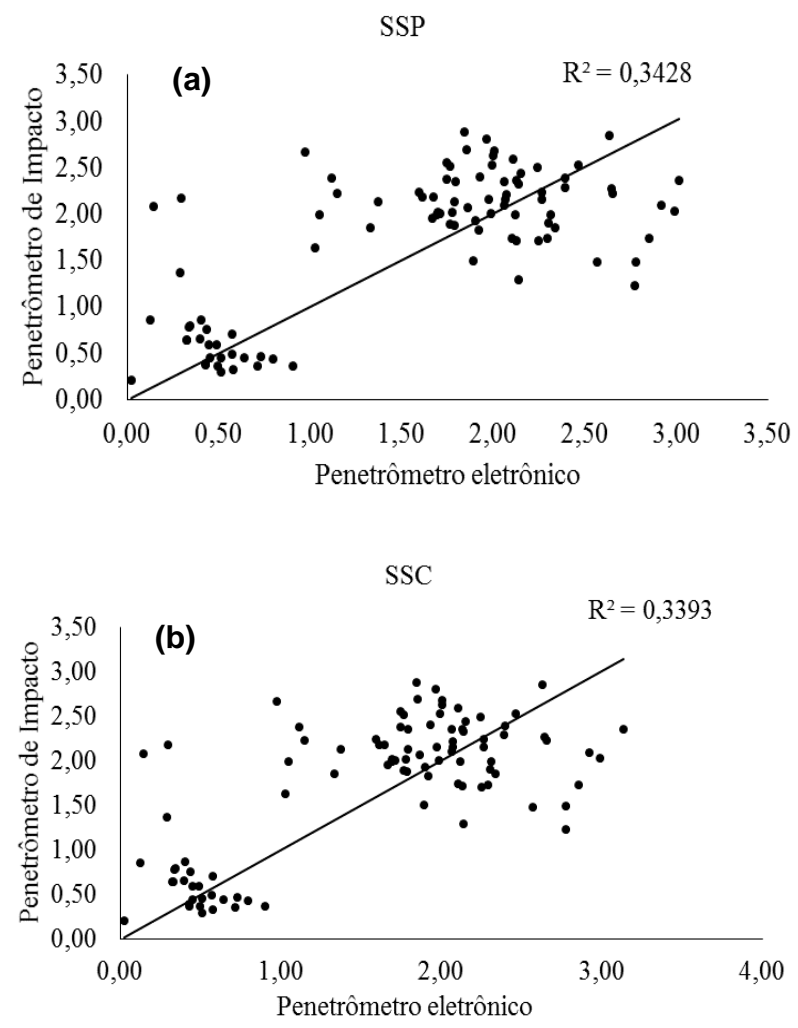

Figura 1. Correlação entre os valores de resistência do solo à penetração determinados com o penetrômetro de impacto e eletrônico no sistema de semeadura direta (a) e convencional (b). 


\section{Conclusões}

Os sistemas de condicionamento do solo não influenciaram nos valores de RP.

O penetrômetro de impacto apresentou valores superiores de resistência à penetração.

Obteve-se correlação positiva média entre os penetrômetros tanto para SSD quanto para SSC.

Ambos os penetrômetros "impacto e eletrônico" podem ser utilizados para obtenção da resistência à penetração.

\section{Referências Bibliográficas}

ARSHAD, M.A.; LOWERY, B.; GROSSMAN, B. Physical tests for monitoring soil quality. In: DORAN, J.W.; JONES, A.J. (Ed.). Methods for assessing soil quality. Soil Science Society of America, Madison, n. 49, p. 123-41, 1996.

BEUTLER, A.; CENTURION. J. F.; SILVA, A. P. Atributos físicos do solo e produtividade da soja após um ano de integração lavoura-pecuária em área sob plantio direto. Revista Engenharia Agrícola, Jaboticabal-SP, v.27, n.1, p.146-151, 2007.

CAIRES, E. F.; FELDHAUS, I. C.; BARTH, G.; GARBUIO, F. J. Lime and gypsum application on the wheat crop. Revista Scientia Agricola, Piracicaba-SP, v. 59, n. 2, p. 357-364, 2002.

CAIRES, E. F.; KUSMAN, M. T.; BARTH, G.; GARBUIO, F. J.; PADILHA, J. M. Alterações químicas do solo e resposta do milho à calagem e aplicação de gesso. Revista Brasileira de Ciência do Solo, Viçosa-MG, v. 28, n. 1, p. 125-136, 2004.

CAMARGO, O. A.; ALLEONI, L. R. F. Compactação do solo e desenvolvimento das plantas. Piracicaba-SP: Esalq, 1997. 132p.

CARVALHO, J. J.; MASIERO, F. C.; LANÇAS, K. P.; RODRIGUES, E. C. P. Avaliação da resistência do solo sob dois sistemas de manejo: plantio direto e convencional. Revista Científica Eletrônica de Agronomia, Garça-SP, v. 22, n. 2, p. 1-11, 2012.

CASTRO, M. A.; CUNHA, F. F.; MAGALHÃES, F. F.; CRUZ, G. H. M. Atributos físico-hídricos do solo ocupado com pastagem degradada e floresta nativa no Cerrado SulMato-Grossense. Brazilian Geographical Journal, ItuiutabaMG, v. 3, n. 2, p. 498- 512, 2012.

CORTEZ, J. W.; ALVES, A. D. S.; MOURA, R. D.; OLSZEVSKI, N.; NAGAHAMA, H. J. Atributos físicos do Argissolo Amarelo do semiárido nordestino sob sistemas de preparo. Revista Brasileira de Ciência do Solo, Viçosa-MG, v. 35, n.4, p. 1207-1216, 2011.

FOLONI, J. S. S.; CALONEGO, J. C.; DE LIMA, S. L. Efeito da compactação do solo no desenvolvimento aéreo e radicular de cultivares de milho. Revista Pesquisa Agropecuária Brasileira, Brasília-DF, v. 38, n. 8, p. 947-953, 2003.

FREDDI, O. S., CARVALHO, M. P., VERONESI JÚNIOR, V.; CARVALHO, G. J. Produtividade do milho relacionada com a resistência mecânica à penetração do solo sob preparo convencional. Revista Engenharia Agrícola, Jaboticabal-SP, v. 26 n.1, p.113-121, 2006

HERRICK, J. E. JONES, T. L. A dynamic cone penetrometer for measuring soil penetration resistance. Soil Science Society of America Journal, Madison, v. 66, n.4, p. 1320-1324, 2002.

LEITE, F., SANTOS, J. E. G. D.; LANÇAS, K. P. Comparação da resistência do solo à penetração entre penetrômetro elétrico-eletrônico e penetrógrafo manual. Revista Cultivando o Saber, Cascavel-PR, v. 3, n. 1, p. 3239, 2010.

LIMA, R. P., LEÓN; M. J.; SILVA, A. R. Comparação entre dois penetrômetros na avaliação da resistência mecânica do solo à penetração. Revista Ceres, Viçosa-MG, v. 60, n. 4, p. $577,2013$.

MAZURANA, M.; LEVIEN, R.; MULLER, J.; CONTE, O. Sistemas de preparo de solo: alterações na estrutura do solo e rendimento das culturas. Revista Brasileira de Ciência do Solo, Viçosa-MG, v. 35, n. 4, p. 1197-1206, 2011.

MÜLLER, M. M. L.; TORMENA, C. A.; GENÚ, A. M.; KRAMER, L. F. M.; MICHALOVIEZ, L.; CAIRES, E. F. Structural Quality of a No-Tillage Red Latosol 50 Months After Gypsum Aplication. Revista Brasileira de Ciência do Solo, Viçosa-MG, v. 36, n. 3, p. 1005-1013, 2012.

PACHECO, E. P.; CANTALICE, J. R. B. Análise de trilha no estudo dos efeitos de atributos físicos e matéria orgânica sobre a compressibilidade e resistência à penetração de um Argissolo cultivado com cana-de-açúcar. Revista Brasileira de Ciência do Solo, Viçosa-MG, v. 35, n. 2, p. 417-428, 2011.

PEDROTTI, A.; PAULETTO, E. A.; CRESTANA, S Resistência mecânica à penetração de um Planossolo submetido a diferentes sistemas de cultivo. Revista Brasileira de Ciência do Solo, Viçosa-MG, v. 25, n.3, p. 521-529, 2001.

RALISCH， R.; MIRANDA， R. M.; OKUMURA， R. S.; BARBOSA, G. M. C.; GUIMARÃES, M. F. SCOPEL, E.; BALBINO, L. C. Resistência à penetração de um Latossolo VermelhoAmarelo do Cerrado sob diferentes sistemas de manejo. Revista Brasileira Engenharia Agrícola Ambiental, Campina Grande-PB, v. 12, n.4, p. 381-384, 2008.

ROBOREDO, D.; MAIA, J. C. S.; OLIVEIRA, O. J. DE; ROQUE, C. G. Uso de dois penetrômetros na avaliação da resistência mecânica de um Latossolo Vermelho distrófico. Revista Engenharia Agrícola, Jaboticabal-SP, v. 30, n. 2, p. 308-314, 2010.

ROCHA, A. T.; OLIVEIRA, A. C.; RODRIGUES, A. N.; LIRA JÚNIOR, M.; FREIRE, F. J.; Emprego do gesso do Araripe na melhoria do ambiente radicular da cana-de-açúcar. Revista Brasileira de Ciências Agrárias, Recife-PB, v. 3, n. 4, p. 307-312, 2008.

ROQUE, C. G.; CENTURION, J. F.; ALENCAR, G. V.; BEUTLER, A. N.; PEREIRA, G. T.; ANDRIOLI, I. Comparação de dois penetrômetros na avaliação da resistência à penetração de um Latossolo Vermelho sob diferentes usos. Revista Acta Scientiarum: Agronomy, Maringá-PR, v. 25, n.1, p. 53-57, 2003

SANTOS, R. L.; FREIRE, F. J.; AZEVEDO, V. M.; ROCHA, A. T.; TAVARES, J. A. Produção de capim elefante e 
movimentação de cátions em função de gesso mineral. Revista Brasileira de Engenharia Agrícola e Ambiental, Campina Grande-PB, v.17, n. 10, p.1030-1037, 2013.

SOUZA, E. B.; PATROCÍNIO FILHO, A. P.; PIMENTA, W. A.; NAGAHAMA, H. J.; CORTEZ, J. W. Resistência mecânica do solo à penetração em função da sua umidade e do tipo de penetrômetro. Revista Engenharia na agricultura, Viçosa-MG, v.22 n.1, p.67-76. 2014.
TAVARES FILHO, J.; BARBOSA, G. M. C.; GUIMARÃES, M. F.; FONSECA, I. C. B.; Resistência do solo à penetração e desenvolvimento do sistema radicular do milho (Zea mays) sob diferentes sistemas de manejo em um Latossolo Roxo. Revista Brasileira de Ciência do Solo, Viçosa-MG, v.25, n.3, p.725-730, 2001.

TORMENA, C. A.; ROLOFF, G. Dinâmica da resistência à penetração de um solo sob plantio direto. Revista Brasileira de Ciência do Solo, Viçosa-MG, v. 20, n. 2, p. 333-39, 1996. 